\title{
From Software Engineering to Business Engineering: ESPRIT Projects in Information Systems Engineering
}

\author{
Pericles Loucopoulos \\ (Invited speaker) \\ Information Systems Group, Department of Computation \\ UMIST, P.O. Box 88 \\ Manchester M60 1QD, UK
}

\begin{abstract}
During this talk it will be argued that approaches to the development and evolution of information systems need to progress from the traditional 'information viewpoint' to also encompass the 'enterprise viewpoint'. It will be argued that future development paradigms will need to provide advanced support to business system analysis in developing, maintaining and evolving high level, formal conceptual models for business applications. To this end, a number of research projects, in the context of ESPRIT, will be discussed and a number of future research directions will be outlined.
\end{abstract}

Pericles Loucopoulos is a professor of Information Systems in the Department of Computation, the University of Manchester Institute of Science and Technology (UMIST). He joined UMIST in 1984 after several years of commercial work in the systems department of a major firm of financial brokers. He holds a Ph.D. in Computing and is a Fellow of the British Computer Society. His research interests include system development methods, rule-based active database environments and requirements engineering. This work has been supported by awards funded by SERC under the Alvey initiative and by the Commission of the European Communities under the ESPRIT and AIM Research and Development programmes. He is the co-author of 3 textbooks and the author and co-author of over 50 papers. 